\title{
The Multi-target Localization Algorithm via Compressed Sensing
}

\author{
Nan Xue and Yan $\mathrm{Hu}$ \\ College of Electrical \& Electronic Engineering, Harbin University of Science and \\ Technology, China \\ xuenan_hrbust@163.com,huyanzjl_001@163.com
}

\begin{abstract}
A multi-target localization algorithm based on compressed sensing was proposed in this paper. The issue of multi-target localization was transformed into compressed sensing. The algorithm greatly reduced the amount of wireless network's communication data by transferring most of the computing work to the central server. This method made full uses of the priori information of the signal and the support set. It dombined Kalman filter with Bayesian compressed sensing to improve the localization accuracy and noise immunity. Simulation results showed that the proposed method has good noise immunity, robustness and localization accuracy compared with traditional locolization methods.
\end{abstract}

Keywords: Compressed Sensing; Bayesian freme; Multi-target localization

\section{Introduction}

Nowadays, there is a growing interest in location based service according to the increasing demand of human location information. GPS location technique is one of the most popular technologies. However, other location techniques must be considered to meet the requirements, because in some special places the GPS signal can't be received or GPS location do not allow to use Since the received signal strength (RSS) localization technology has the features of simple equipment, low cost, easy to implement, and thus become the most widely used localization technology The major methods of RSS location include geometric localization method based on path loss model and the location fingerprint matching method. Compared to the former, the latter has the characteristics of high precision, strong robustness, etc.

Using location fingepprint matching algorithm, location area is divided into many grids, thus location fingerprint leature is represented by mean value of grid center' signal strength, and deterministic reasoning algorithm is applied to calculate the location of the target. Compare with the number of mesh network, the number of mobile target is very few, which satisfy the sparsity condition of compressed sensing [1-3]. At the same time, only a small amount of observation data can accurately reconstruct the original signal by using compressed sensing technology, therefore the study of applying compressed sensing to the problem of wrieless network location becomes an academic focus in recent years. There are two advantages of applying compressed sensing to wireless network location. First, without additional complex compression algorithm, sensors only need to collect a small amount of observation data, and accomplish sampling as well as compression at the meantime. Second, the harsh constraint of energy and computing capability of sensor nodes is avoided by transferring signal reconstruction process to the central server.

Feng et al., [4] use compressed sensing to lower sampling rate so that a few observations can finish location process. However, this model has low precision and poor anti-noise ability 
due to BP algorithm used as the recovery algorithm. Nikitaki et al., [5] sample signal strength of each location node, then reconstruct the signal by 1 - norm minimization. This model can accurately locate the target without noise, but, in the presence of noise, reconstruction is not ideal, the complexity of the algorithm is high, and real-time is not good as well. In addition, endurance of wireless network is also reduced because wireless locating process is carried out at the terminal whose energy and calculation, as well as storage ability is limited.

A multi-target localization algorithm (MCL) based on compressed sensing was designed in this paper, the definite system model and operation mode were also included. This MCL algorithm made full uses of the priori information of the signal and the support set, reduced computational complexity and improved real-time. It combined Kalman filter with Bayesian compressed sensing [6] to improve the localization accuracy and noise immunity. The algorithm greatly reduced the amount of wireless network's communication data by transferring most of the computing work to the central server. Simulation results strowed that the proposed method has good noise immunity, robustness and localization accuracy compared with traditional localization methods.

\section{Localization Model based on Compressed Sensing}

\subsection{Compressed Sensing}

Compressed sensing is a signal processing technique for efficiently acquiring and reconstructing a signal, by finding solutions to under-determined linear systems. This takes advantage of the sparseness or compressibility of the signal in some domain, allowing the entire signal to be determined from relatively few neasurements.

In the theory of compressed sensmg, using the basis matrix $\Psi \in R^{N \times N}$ with the vectors $\{\psi i\}$ as columns, a signal $x \in R^{N}$ can be expressed as

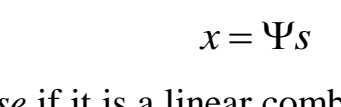

The signal $x$ is $K$-sparse if it is a linear combination of only $K$ basis vectors; that is, only $K$ of the $s_{i}$ coefficients in Equation(1) are nonzero and $(N-K)$ are zero. Assumed that a stable measurement matrix $\Phi \in R^{M \times N} \quad(M<<N)$ can be linearly transformed with $x$, then measurements $y$ can be writtends

$$
y=\Phi x
$$

Then the compressed sensing can be represented as

$$
y=\Phi x=\Phi \Psi s=\Theta s
$$

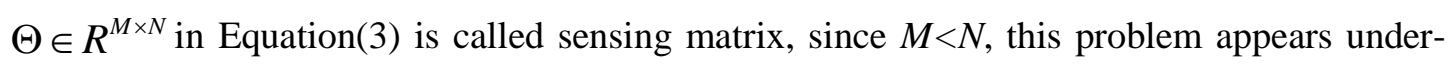
determined. Compressed sensing proves that if $s$ is sparse and sensing matrix $\Theta$ satisfy restricted isometry property, then original signalcan be reconstructed by solving optimal $l_{0^{-}}$ norm

$$
\begin{aligned}
& \min \|s\|_{0} \\
& \text { s.t. } Y=\Phi X=\Phi \Psi s
\end{aligned}
$$




\subsection{Modeling by Compressed Sensing}

The model of location system is showed in Figure 1, where the whole location area is divided into $N$ grid. There are $D$ sensors whose positions are definite using wireless communications with center server, and there are also $K$ mobile targets randomly located on the grid, whose positions are unknown. The quantity of moving targets $K$ is sparse compared with $N$, that is, the quantity of grid $(N>>K)$. This sparsity provides theoretical guarantee for compressed sensing applied to the location system. In this localization system, the sensors periodically collect signal strength of mobile targets, transmit the accumulated signal strength of the moving targets to the central server in one cycle. Using MCL algorithm, the center server compares signal strength sent by sensors with signal strength stored in location fingerprint database to determine which grid the moving target is in.

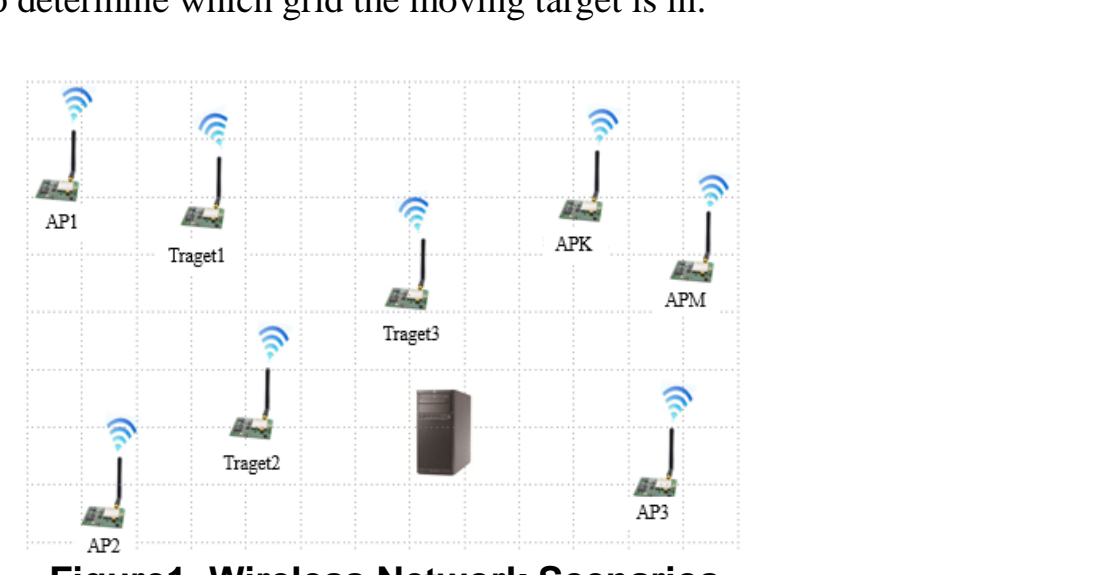

Figure1. Wireless Network Scenarios

Therefore a fingerprint database of signal strength is necessary to locate. Signal strength in each grid of Mobile targets in location area is collected by sensor, all signal strength vectors collected in $\mathrm{N}$ grid are combined as a matrix $\Psi$, that is

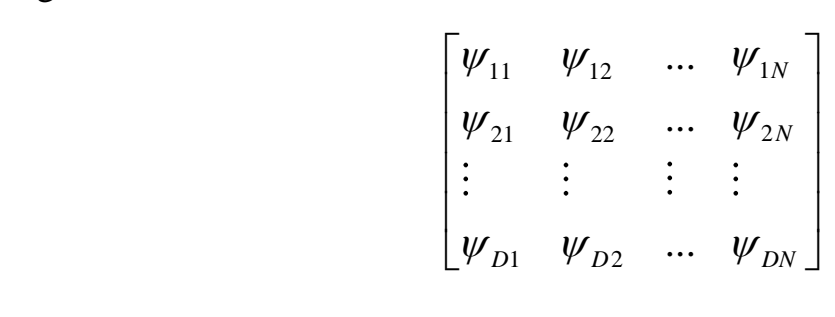

Where $\psi_{d, n}$ is mobile target' signal strength in the $n$th grid received by the $d$ th sensor. Sensors transmit the accumulated signal strength of the moving targets to the central server, where signal strength is combined to signal strength fingerprint database $\Psi$ according to the position. In the environment where does not changed obviously, location fingerprint database can be served as a standard library used repeatedly by just single measurement.

In Jocation process, signal strength of mobile targets in location area collected by each sensor will be transmitted to the central server. Central server uses a random Gaussian measurement matrix $\Phi \in R^{M \times D}$ to complete the process of compressive sampling. The signal value $x_{i}$ represents the sum of RSS signal strength received by the $i$ th sensor. RSS signal strength $x_{i}$ can be seen as the linear combination of a few column vectors of location fingerprint $\Psi$. Furthermore, $x_{i}$ can be expressed as the product of location fingerprint $\Psi$ and the location signal vector. Signal $x$ can be expressed as 


$$
\left[\begin{array}{l}
x_{1} \\
x_{2} \\
\vdots \\
x_{D}
\end{array}\right]=\left[\begin{array}{llll}
\psi_{11} & \psi_{12} & \ldots & \psi_{1 N} \\
\psi_{21} & \psi_{22} & \ldots & \psi_{2 N} \\
\vdots & \vdots & \vdots & \vdots \\
\psi_{D 1} & \psi_{D 2} & \ldots & \psi_{D N}
\end{array}\right]\left[\begin{array}{l}
s_{1} \\
s_{2} \\
\vdots \\
s_{N}
\end{array}\right]
$$

Signal $x$ can be written as

$$
x=\Psi s
$$

The process of sampling and compression is written as

$$
y=\Phi x=\Phi \Psi s=\Theta s
$$

Because of noise interference of actual location environment, Equation(7) can be expressed as

$$
y=\Phi x+E=\Phi \Psi s+E=\Theta s+E
$$

Where $E$ is the measurement noise, $s$ indicates the position signal vector to be recovered, the sample matrix $\Phi$ is a random Gauss matrix, and fingerpunt database $\Psi$ is a fixed matrix obtained from measurement. Because of strong correlation between the sampling matrix $\Phi$ and the fingerprint database, there is a theoretical possibility to accurately reconstruct the original signal by using compressed sensing.

The problem of multi-target localization based on signal strength converts into compressed sensing problem, which reconstruct sparse vectors according to the measurement results. Using MCL algorithm designed in this paper, the solution can be achieved.

\subsection{Multi-target CS Localization Algorithm}

The localization algorithmin this paper combined Kalman filter with Bayesian compressed sensing to improve the rocalization accuracy and noise immunity. MCL algorithm, which improved based on Kalman filtered compressed sensing [7, 8], firstly obtain the residuals of observations compressed signal and then estimate the position of signal residual mutation by Bayesian ompression, through this method to resume the position information vector $s$. 1. Initialization al gorithm

In $t=0, y_{0}$ is the Nalue which compressed by $\Phi, y=y_{0}$, support set is $\tilde{T}_{0}, \hat{s}_{0}$ is the value estimate by Bayesian compressed sensing. When $t=0$, Bayesian compressed sensing is used to solve Equation(9). Solving process is as follows.

In Equation( $(2), E$ is Gaussian white noise, assumed the variance is $\sigma_{0}^{2}$, in the Bayesian framework likelihood function of position signal vector $s$ and Gaussian noise variance $\lambda_{0}$ $\left(R_{0}-1 / \sigma_{0}^{2}\right)$ can be obtained.

$$
P\left(y \mid \mathrm{s}, \lambda_{0}\right)=\left(2 \pi / \lambda_{0}\right)^{-M / 2} \exp \left(-\frac{\lambda_{0}}{2}\|y-\Theta s\|_{2}^{2}\right)
$$

In the Bayesian framework, vector $s$ is sparse means that it can contribute to their obedience to a sparse prior distribution, a common distribution is the Laplace density distribution, in this paper, a common hierarchical sparse priori is endued to the original signal. 


$$
P(s \mid \lambda)=\prod_{j=1}^{N} N\left(s_{j} \mid 0, \lambda_{j}^{-1}\right)
$$

In Equation(11), $N\left(\cdot \mid 0, \lambda_{j}^{-1}\right)$ is Gaussian distribution with zero mean and $\lambda_{j}^{-1}$ variance. With Equation(10) and Equation(11), posterior probability distribution of $s$ can be obtained.

$$
\begin{aligned}
& P\left(s \mid y, \lambda, \lambda_{0}\right)=\frac{P\left(y \mid s, \lambda_{0}\right) P(s \mid \lambda)}{P\left(y \mid \lambda, \lambda_{0}\right)} \\
& =(2 \pi)^{-\frac{N+1}{2}}|\Sigma|^{-\frac{1}{2}} \exp \left\{-\frac{1}{2}(s-\mu)^{T}(\Sigma)^{-1}(s-\mu)\right\}
\end{aligned}
$$

$s$ is Gaussian distribution with $\sum$ mean and $\mu$ variance, they equal to

$$
\begin{aligned}
& \mu=\lambda_{0} \sum \Theta^{T} y \\
& \Sigma=\left(\lambda_{0} \Theta^{T} \Theta+\Lambda\right)^{-1} \\
& \Lambda=\operatorname{diag}\left(\lambda_{1}, \lambda_{2}, \ldots, \lambda_{N}\right)
\end{aligned}
$$

To get value of mean $\sum$ and variance $\mu$, we should know hyperparameter $\lambda$ and $\lambda_{0}$ firstly, $\lambda$ and $\lambda_{0}$ can be derived by maximizing the logarithm marginal likelihood

$$
\begin{aligned}
& L\left(\lambda, \lambda_{0}\right)=\log \int P\left(y \mid s, \lambda_{0}\right) P(s \mid \lambda) d s=\frac{1}{2}\left[M \log 2 \lambda+\log |C|+y^{T} C^{-1} y\right] \\
& \left.C=\lambda_{0}{ }^{-1} I+\Theta \Lambda^{-1} \Theta^{T} \text { in Equation } 4\right) \text {. }
\end{aligned}
$$

In the framework of Relevance Vector Máchine ${ }^{[9,10]}, \lambda$ and $\lambda_{0}$ can be estimated as following

$$
\text { Q } 1 / \lambda_{0}{ }^{\text {new }}=\frac{\|y-\Theta \mu\|_{2}^{2}}{M-\sum_{i} \gamma_{i}}
$$

The convergence appears by iterating between Equations(13), (15) and (16), vector $s$ can be represented by $\mu$.

When $t>1$, Kalman filter measurement equation is Equation(9), equations of state is

$$
s_{t}=s_{t-1}+w_{t-1}
$$

through Kalman filter,residuals $\tilde{y}_{t, \text { res }}$ can be obtained, solving process is as follows

$$
\begin{gathered}
P_{t \mid t-1}=P_{t-1}+\hat{Q}_{t} \\
K_{t}=P_{t \mid t-1} \Theta^{\prime}\left(\Theta P_{t \mid t-1} \Theta^{\prime}+\sigma^{2} I_{T}\right)^{-1} \\
\hat{s}_{t}=\left(I-K_{t} \Theta\right) \hat{s}_{t-1}+K_{t} y_{t}
\end{gathered}
$$

$$
\tilde{y}_{t, r e s}=y_{t}-\Phi \hat{x}_{t}
$$


2. Residuals estimate.

Equation(4) is substituted into Equation(21)

$$
\tilde{y}_{t, r e s}=\Theta\left(s_{t}-\hat{s}_{t}\right)=\Theta \beta_{t}
$$

$\beta_{t}=s_{t}-\hat{s}_{t}$ is the part of the signalchanges.

3. Reconstruction of the location information signal.

Bayesian compressed sensing algorithms is used to reconstruct the residual through Equation(22). If $\hat{\beta}_{t}$ is the recovery result of $\beta_{t}$, the formula is achieved as following

$$
\tilde{s}_{t}=\hat{s}_{t}+\hat{\beta}_{t}
$$

$\tilde{s}_{t}$ is result modified by Bayesian compressed sensing.For the next time signal estimation, $P_{t}$ and $\tilde{s}_{t}$ are simply used to replace $P_{t-1}$ and $\hat{s}_{t-1}$ in Equation(18) and Equation(19) to complete the update.

\section{The Simulation Results Analysis}

\subsection{Localization Results of MCL Algorithm}

Simulation scenario is $20 \mathrm{~m} \times 20 \mathrm{~m}$ indoor area-and is divided inte the size of $1.0 \mathrm{~m} \times 1.0 \mathrm{~m}$ grid. The number of the moving object is $K \cong 10$, randomly distributed in the room, the number of observations $M=40$. As shown in Figure 2, the abscissa axis represents the horizontal position, the vertical axis represents the vertical position, the red asterisk represents the original location of the mobile terminal in the region, through the black circles represent the position of MCL algorithm recoyered after the terminal when the SNR $=15 \mathrm{~dB}$. Simulation results show that the original location and recovery location can completely overlap when $\mathrm{SNR}=15 \mathrm{~dB}$, it proved that the proposed algorithm in the presence of noise and still accurate recovery ignal.

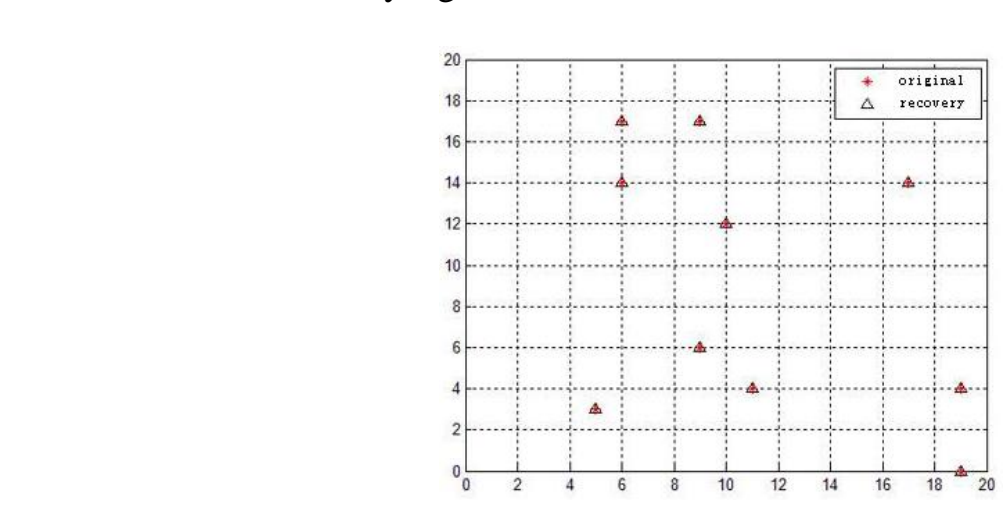

Figure 2. Localization Results of MCL

\subsection{Reconstruction Error Comparison of KNN, BP, KFCS and MCL}

Figure 3 is reconstruction error comparison of KNN, BP, KFCS and MCL under different noise level. Simulation results show that the proposed MCL method performs much better than the other traditional methods, especially in the high noise environment. 


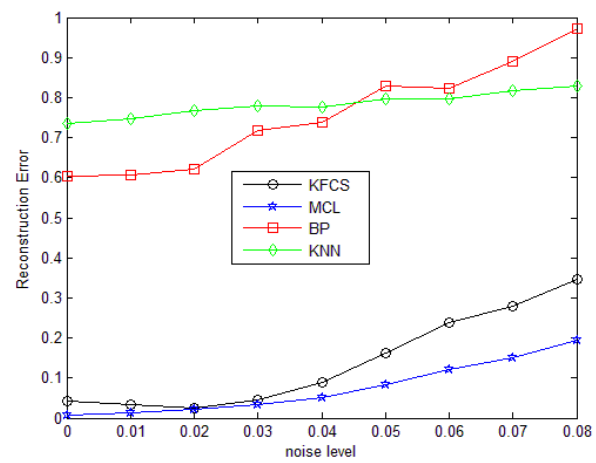

\section{Figure 3. Reconstruction Error Comparation of KNN, BP, KFCS and MCL}

\section{Summary}

MCLlocalization algorithm converted wireless sensor network ocalization into a compressed sensing problem, transferred the main computational to the central localization server, reduced energy consumption and storage capacity, improved the efficiency of the localization system. MCL algorithm combined Kalman filter and Bayesian compressed sensing, make full use of the prior information of signals to improve localization accuracy and noise immunity. Simulation results show that MCL location algorithm performance well in noisy environment.

\section{References}

[1] D. Donoho, "Compressed sensing", IEEE Transactions on Information Theory, vol. 52, (2006), pp. 12891306.

[2] E. Candès, "Compressive sampling", Proceedings or the Inter-national Congress of Mathematicians, (2006), 3, pp. 1433-1452.

[3] E. Candès, J. Romberg and T. Tao, "Robust uncertainty principles: exact signal reconstruction from highlyincomplete frequency information", IEEE Transactions on Information Theory, vol. 52, (2006), pp. 48.

[4] C. Feng, S. Valaee and Z. H. Tan, "Multiple Target Localization Using Compressive Sensing", IEEE Global Telecommunications Conference, GLOBECOM, (2009), pp. 1-6.

[5] S. Nikitak and P. Tsakalides "Zocalizationin wireless networks via spatial sparsity", Conference Record of the Forty Fourtb Asilomar Conference on Signals, Systems and Computers, (2010),pp. 236-239.

[6] S. Ji, Y. Xue and L .Carin "Bayesian compressive sensing", IEEE Transactions on Signal Processing, vol. 56, (2008), pp. 2346-2356.

[7] N. Vaswani, "Kr-CS: Compressive Sensing on Kalman Filtered Residual”, http:\|www.computing.edu.au/ dsp/cs.

[8] N. Vaswani, “Analyzing least squares and kalman filtered compressed sensing”,IEEE International Conference onAcoustics, Speech and Signal Processing, (2009), pp. 3013-3016.

[9] A. Maro and T. Figueiredo, "Adaptive sparseness using Jeffreys prior", Advances in Neural Information Processing Systems (NIPS14), (2002), pp. 697-704.

[10] M. E.Tpping, "Sparse Bayesian learning and the relevance vector machine", Journal of Machine Learning Research, vol. 1, (2001), pp.211-244. 
International Journal of Multimedia and Ubiquitous Engineering Vol. 9, No. 11 (2014)

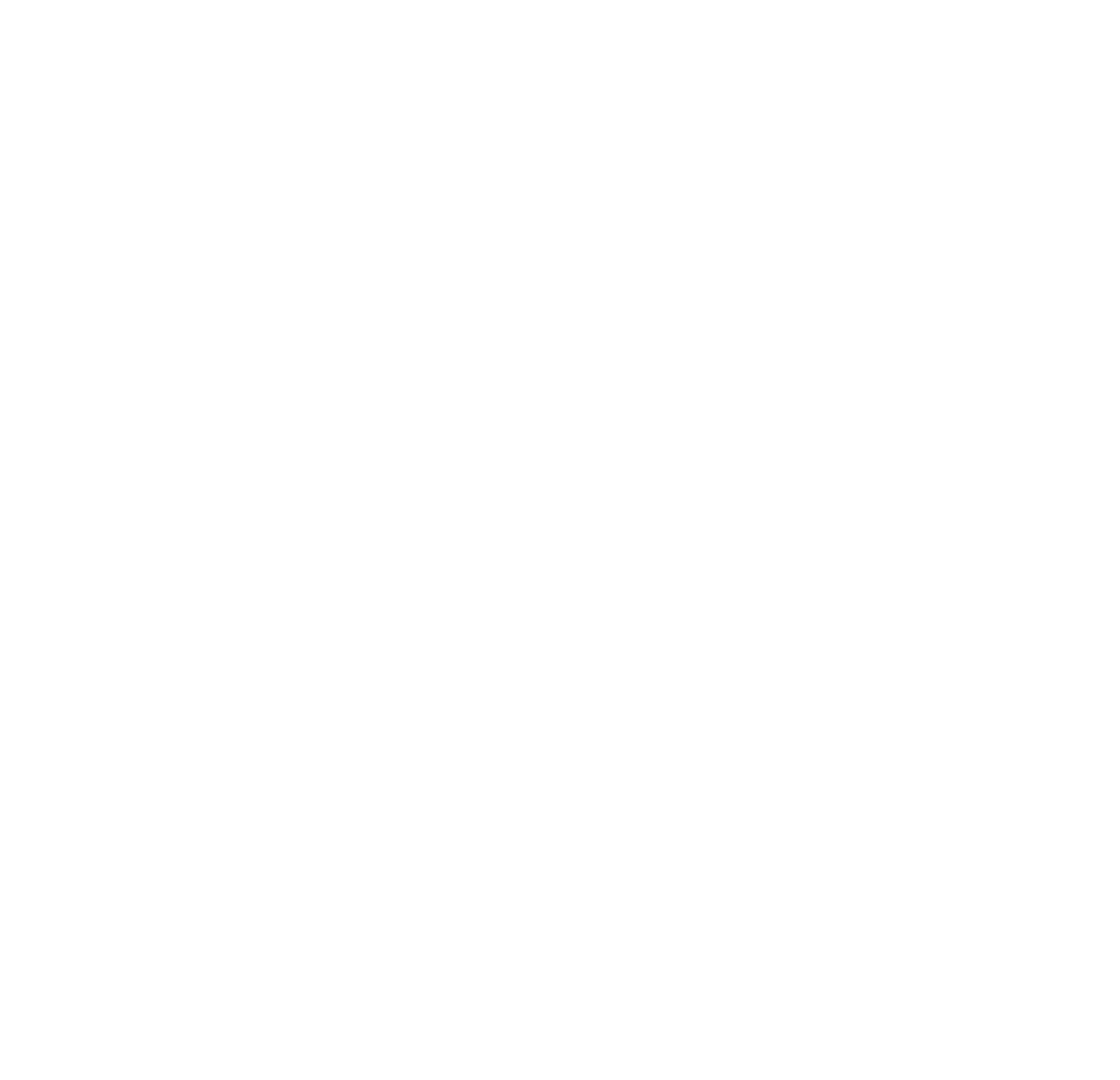

